\title{
THF Hydrates as Model Systems for Natural Gas Hydrates: Comparing their Mechanical and Vibrational Properties
}

\author{
Thomas M. Vlasic, Phillip D. Servio, Alejandro D. Rey* \\ Department of Chemical Engineering, McGill University, Montréal, QC, H3A 0C5 \\ *e-mail: alejandro.rey@mcgill.ca; tel. : 514-398-4196
}

SI 1. Equation of state fitting

Vinet isothermal equation of state:

Energy-explicit form:

$\Delta E(V)=\frac{4 V_{0} B_{0}}{\left(B_{0}^{\prime}-1\right)^{2}}-\frac{4 V_{0} B_{0}}{\left(B_{0}^{\prime}-1\right)^{2}}\left[1-\frac{3}{2}\left(B_{0}^{\prime}-1\right)\left(1-\left(\frac{V}{V_{0}}\right)^{1 / 3}\right)\right] \exp \left[\frac{3}{2}\left(B_{0}^{\prime}-1\right)\left(1-\left(\frac{V}{V_{0}}\right)^{1 / 3}\right)\right]$

Pressure-explicit form:

$P(V)=3 B_{0} \frac{\left[1-\left(\frac{V}{V_{0}}\right)^{1 / 3}\right]}{\left(\frac{V}{V_{0}}\right)^{2 / 3}} \exp \left[\frac{3}{2}\left(B_{0}^{\prime}-1\right)\left(1-\left(\frac{V}{V_{0}}\right)^{1 / 3}\right)\right]$

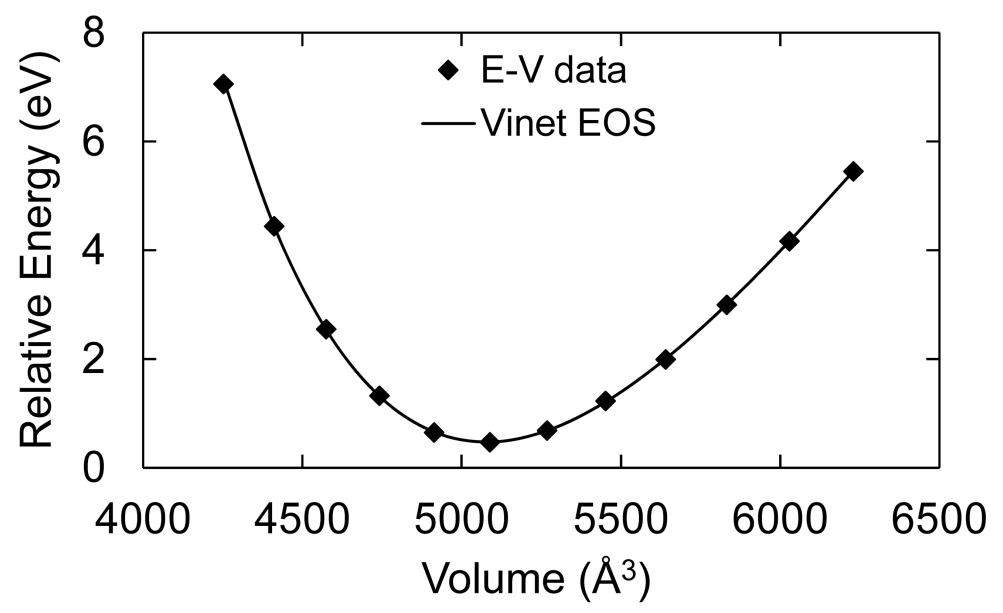

Figure S1. Fitting the Vinet EOS to the energy-volume data for THF sll hydrates. 


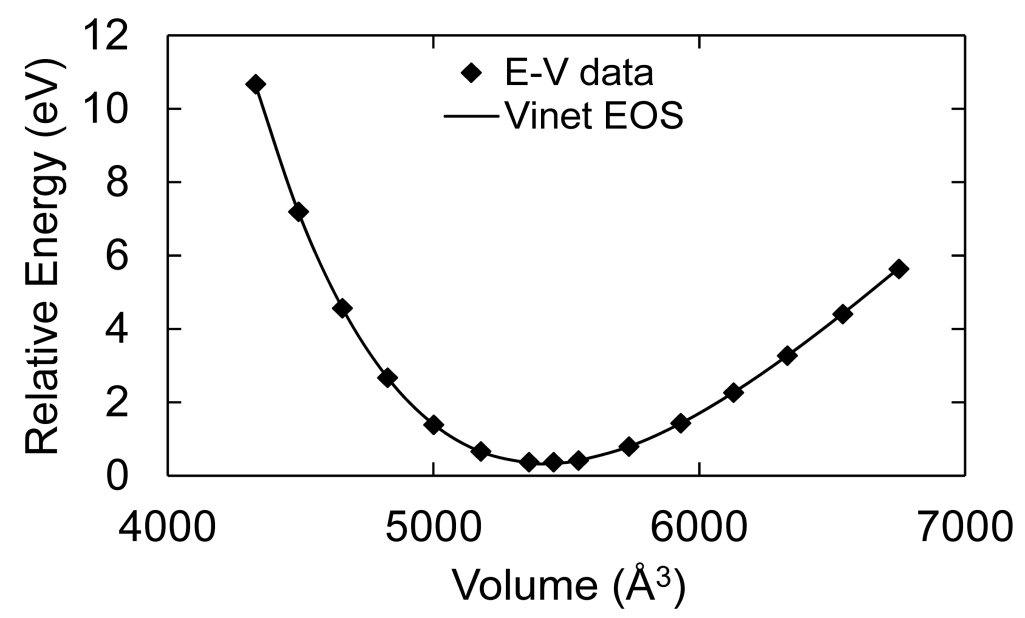

Figure S2. Fitting the Vinet EOS to the energy-volume data for THF-Xe sll hydrates.

SI 2. Unprocessed IR spectra
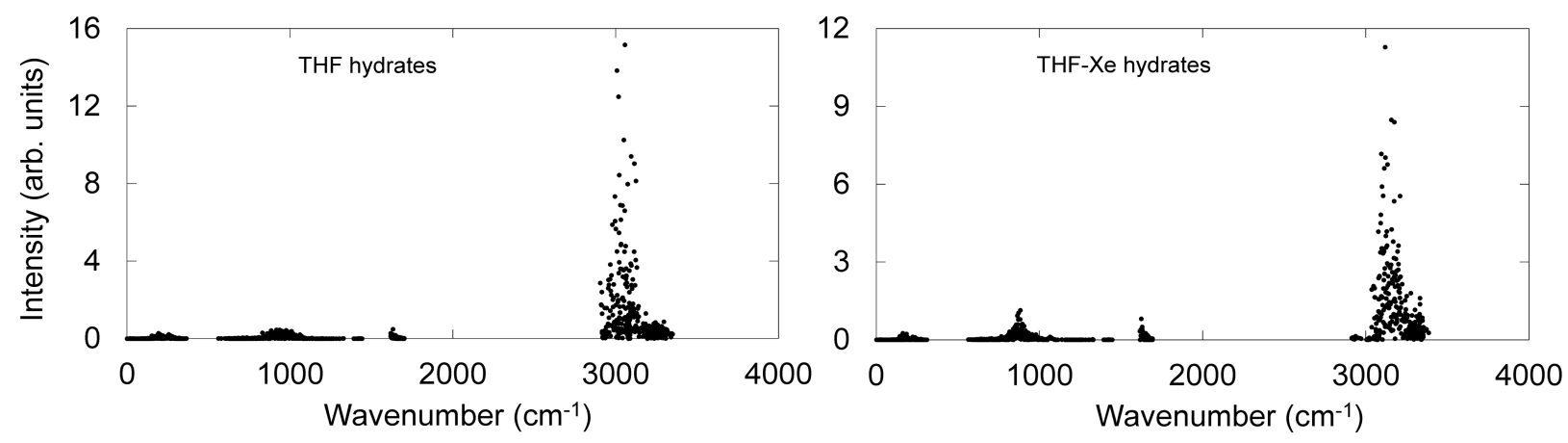

Figure S3. Raw IR data for THF and THF-xenon sll hydrates.

SI 3. Monocrystalline elastic constants

Table S1. Hydrocarbon gas hydrate literature values for single-crystal cubic elastic constants, Zener $\left(A_{z}\right)$ and Every $\left(A_{e}\right)$ anisotropy factors.

\begin{tabular}{|c|c|c|c|c|c|}
\hline Hydrate structure & $c_{11}(\mathrm{GPa})$ & $C_{12}(\mathrm{GPa})$ & $C_{44}(\mathrm{GPa})$ & $A_{z}$ & $\overline{A_{e}}$ \\
\hline $\mathrm{C}_{3} \mathrm{H}_{8}$ sll, theo. ${ }^{a}$ & 16.2 & 8.6 & 4.8 & 1.25 & -0.16 \\
\hline$i-\mathrm{C}_{4} \mathrm{H}_{10} \mathrm{sll}$, theo. ${ }^{a}$ & 14.9 & 7.2 & 4.7 & 1.23 & -0.18 \\
\hline $\mathrm{C}_{2} \mathrm{H}_{6}-\mathrm{CH}_{4}$ sll, theo. ${ }^{a}$ & 16.9 & 6.1 & 6.1 & 1.13 & -0.13 \\
\hline $\mathrm{C}_{3} \mathrm{H}_{8}-\mathrm{CH}_{4}$ sll, theo. ${ }^{a}$ & 15.7 & 6.9 & 5.3 & 1.20 & -0.17 \\
\hline $\mathrm{C}_{3} \mathrm{H}_{8}-\mathrm{CH}_{4}$ sll, theo. ${ }^{b}$ & 15.3 & 7.0 & 4.6 & 1.11 & -0.08 \\
\hline $\mathrm{CH}_{4} \mathrm{sl}$, theo. ${ }^{b}$ & 14.2 & 6.0 & 7.9 & 1.93 & -1.21 \\
\hline
\end{tabular}




\begin{tabular}{|l|c|c|c|c|c|}
\hline $\mathrm{CH}_{4} \mathrm{sl}$, theo. $^{c}$ & 18.1 & 5.7 & 6.2 & 0.99 & 0.0 \\
\hline $\mathrm{CH}_{4}$ sl, exp. ${ }^{d}$ & 11.9 & 6.3 & 3.4 & 1.21 & -0.14 \\
\hline
\end{tabular}

alasic et al. ${ }^{35},{ }^{b}$ Huo et al. ${ }^{44}$, ${ }^{c}$ Jendi et al. ${ }^{37}$, ${ }^{d}$ Shimizu et al. ${ }^{45}$

\section{SI 4. Polycrystalline elastic properties}

Table S2. Relevant hydrate polycrystalline mechanical properties from the literature: lattice constant, $a_{0}(\AA)$, isothermal bulk modulus, $B(G P a)$, shear modulus, G (GPa), Young's modulus, $E(G P a)$, compressional wave velocity, $V_{p}(\mathrm{~km} / \mathrm{s})$, shear wave velocity, $V_{s}(\mathrm{~km} / \mathrm{s})$, Poisson ratio, $v$, and density, $\rho\left(\mathrm{Kg} / \mathrm{m}^{3}\right)$.

\begin{tabular}{|c|c|c|c|c|c|c|c|c|}
\hline Hydrate structure & $a_{0}$ & $B$ & $\bar{G}$ & $E$ & $V_{p}$ & $\mathrm{~V}_{\mathrm{s}}$ & $\bar{v}$ & $\bar{\rho}$ \\
\hline 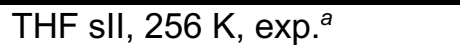 & & 8.27 & 2.65 & 7.17 & 3.513 & 1.663 & 0.355 & \\
\hline THF sll, $273 \mathrm{~K}, \exp ^{b}$ & & 8.5 & 3.5 & & 3.665 & 1.890 & & \\
\hline 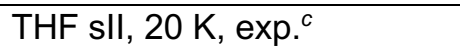 & 17.14 & & & & & & & \\
\hline THF sll, $263 \mathrm{~K} / 274 \mathrm{~K}$, exp. ${ }^{d}$ & & & & & $3.560 / 3.150$ & & & 967 \\
\hline 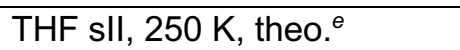 & 17.14 & 10.62 & & & & & & \\
\hline 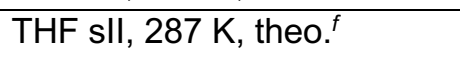 & 17.34 & 6.0 & & & & & & \\
\hline THF-Xe sll, $298 \mathrm{~K}$, exp. ${ }^{g}$ & 17.46 & 8.8 & & & & & & \\
\hline 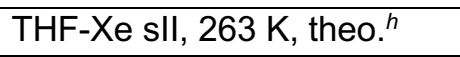 & 17.23 & & & & & & & \\
\hline $\mathrm{C}_{3} \mathrm{H}_{8}$ sll, $0 \mathrm{~K}$, theo. ${ }^{i}$ & 17.09 & 11.14 & 4.36 & 11.57 & 4.263 & 2.162 & 0.327 & 932.9 \\
\hline $\mathrm{C}_{3} \mathrm{H}_{8}$ sll, $287 \mathrm{~K}$, theo. ${ }^{f}$ & 17.27 & 8.96 & & & & & & \\
\hline$i-\mathrm{C}_{4} \mathrm{H}_{10} \mathrm{sll}, 0 \mathrm{~K}$, theo. ${ }^{i}$ & 17.28 & 9.76 & 4.34 & 11.33 & 4.070 & 2.150 & 0.306 & 938.4 \\
\hline $\mathrm{C}_{2} \mathrm{H}_{6}-\mathrm{CH}_{4}$ sll, $273 \mathrm{~K}$, exp. ${ }^{j}$ & & 8.51 & 3.68 & & 3.822 & 2.001 & 0.311 & \\
\hline $\mathrm{C}_{2} \mathrm{H}_{6}-\mathrm{CH}_{4}$ sll, $0 \mathrm{~K}$, theo. ${ }^{i}$ & 17.21 & 9.70 & 5.79 & 14.48 & 4.258 & 2.455 & 0.251 & 960.8 \\
\hline $\mathrm{C}_{3} \mathrm{H}_{8}-\mathrm{CH}_{4} \mathrm{sll}, 0 \mathrm{~K}$, theo. ${ }^{i}$ & 17.33 & 9.90 & 4.81 & 12.42 & 4.088 & 2.220 & 0.291 & 976.1 \\
\hline $\mathrm{C}_{3} \mathrm{H}_{8}-\mathrm{CH}_{4}$ sll, $0 \mathrm{~K}$, theo. ${ }^{k}$ & 17.72 & 9.76 & 4.43 & 11.55 & 4.141 & 2.203 & 0.303 & 910.0 \\
\hline $\mathrm{C}_{3} \mathrm{H}_{8}-\mathrm{CH}_{4}$ sll, $287 \mathrm{~K}$, theo..$^{f}$ & 17.42 & 9.93 & & & & & & \\
\hline $\mathrm{CH}_{4} \mathrm{Sl}, 273 \mathrm{~K}, \exp ^{j}$ & & 8.39 & 3.54 & & 3.766 & 1.957 & 0.315 & 924 \\
\hline $\mathrm{CH}_{4} \mathrm{sl}, 271 \mathrm{~K}$, exp.' & & 9.11 & & & & & & \\
\hline $\mathrm{CH}_{4} \mathrm{sl}, 0 \mathrm{~K}$, theo. $^{k}$ & 12.13 & 9.98 & 4.62 & 12.01 & 4.205 & 2.250 & 0.299 & 910 \\
\hline $\mathrm{CH}_{4} \mathrm{sl}, 0 \mathrm{~K}$, theo. ${ }^{m}$ & 11.90 & 9.82 & 6.23 & 15.4 & 4.386 & 2.571 & 0.238 & 943 \\
\hline
\end{tabular}

${ }^{a}$ Bathe et al. ${ }^{46},{ }^{b}$ Kiefte et al. ${ }^{47},{ }^{c} \mathrm{Tse}{ }^{49}$, dPohl et al. ${ }^{48}$, ${ }^{e}$ Alavi et al. $.{ }^{50},{ }^{f}$ Fang et al $.{ }^{51},{ }^{9}$ Manakov et al. ${ }^{43},{ }^{h}$ Alavi et al. ${ }^{52}$, 'Vlasic et al. ${ }^{35}$, ${ }^{j}$ Helgerud et al. ${ }^{53}$, ${ }^{k}$ Huo et al. ${ }^{44}$, 'Klapproth et al. ${ }^{54},{ }^{m}$ Jendi et al. ${ }^{37}$

Shear modulus:

$$
\begin{gathered}
G_{\text {Reuss }}=\frac{5\left(c_{11}-c_{12}\right) c_{44}}{4 c_{44}+3\left(c_{11}-c_{12}\right)} \\
G_{\text {Voigt }}=\frac{c_{11}-c_{12}+3 c_{44}}{5}
\end{gathered}
$$




$$
G=\frac{G_{\text {Reuss }}+G_{\text {Voigt }}}{2}
$$

Bulk modulus:

$$
B=B_{\text {Reuss }}=B_{\text {Voigt }}=\frac{c_{11}+2 c_{12}}{3}
$$

Poisson's ratio:

$$
v=\frac{\frac{3}{2} B-G}{G+3 B}
$$

Young's modulus:

$$
E=2 G(1+v)
$$

Compressional (longitudinal) wave velocity:

$$
V_{p}=\sqrt{\frac{B+\frac{4}{3} G}{\rho}}
$$

Shear (transverse) wave velocity:

$$
V_{s}=\sqrt{\frac{G}{\rho}}
$$

Zener anisotropy factor:

$$
A_{z}=\frac{2 c_{44}}{c_{11}-c_{12}}
$$

Every anisotropy factor:

$$
A_{e}=\frac{c_{11}-c_{12}-2 c_{44}}{c_{11}-c_{44}}
$$


SI 5. Structure-property relationships

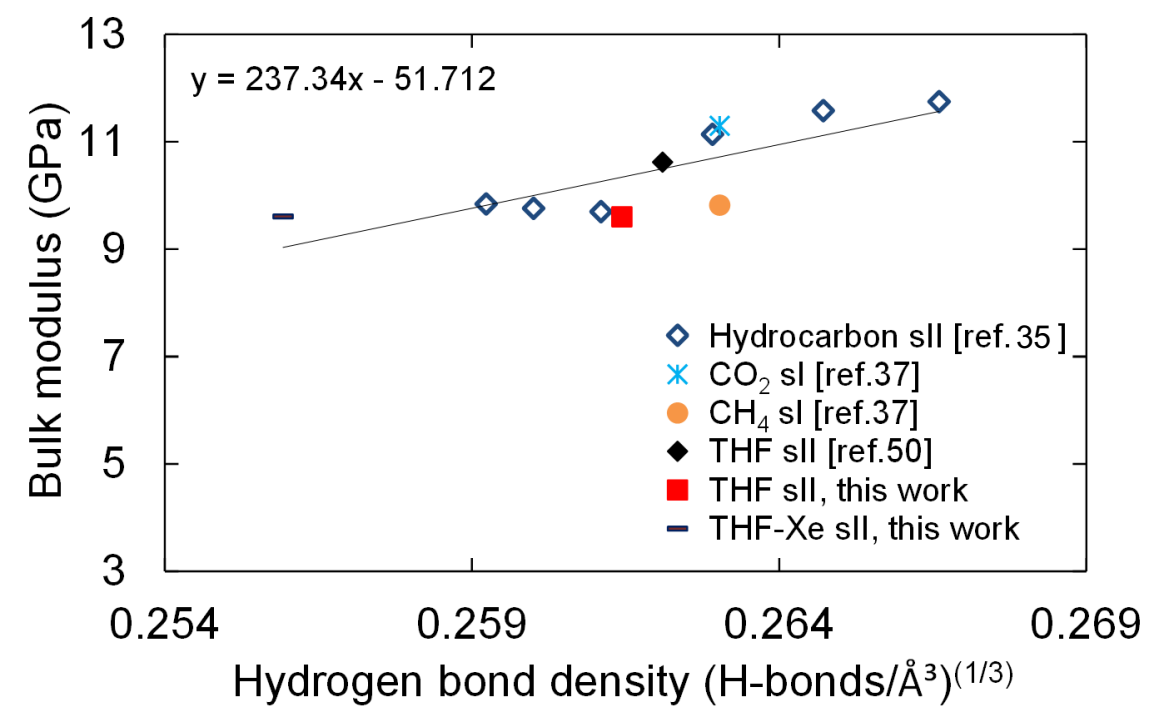

Figure S4. The effect of hydrogen bond density on the bulk modulus for sl and sll hydrates (calculated using the elastic constants method).

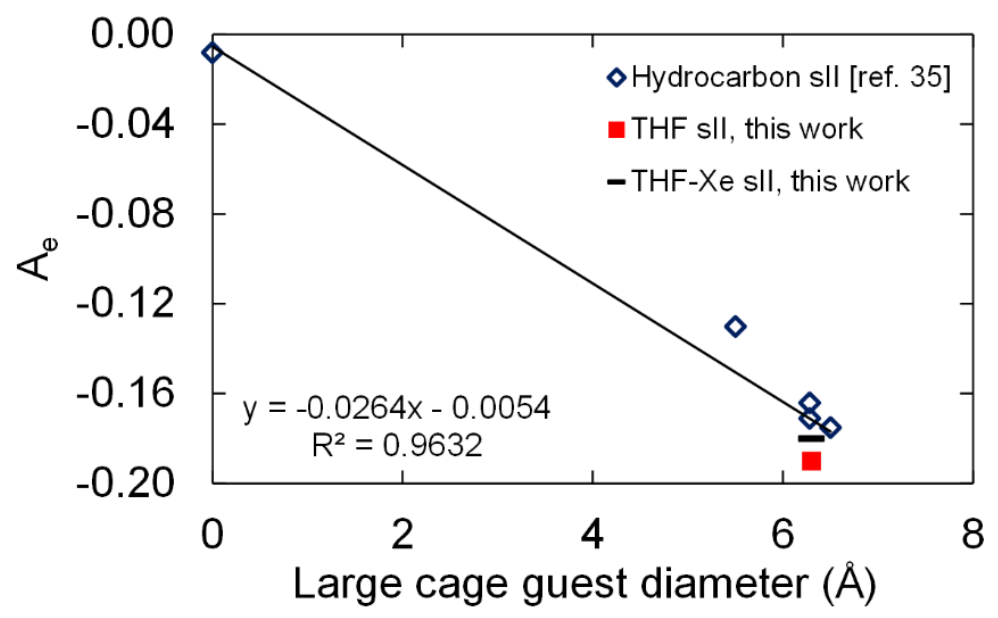

Figure S5. The effect of large cage guest diameter on the Every anisotropy factor $\left(A_{e}\right)$ for sll hydrates. (Note: guest diameters taken from Sloan and Koh $2007^{14}$ ) 


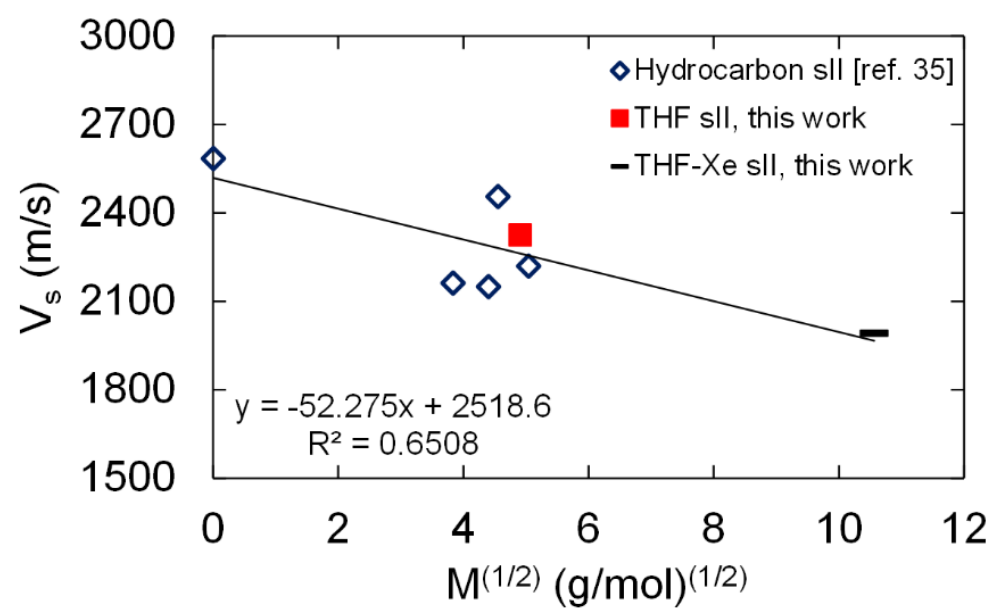

Figure S6. The effect of average guest molecular mass on the shear wave velocity.

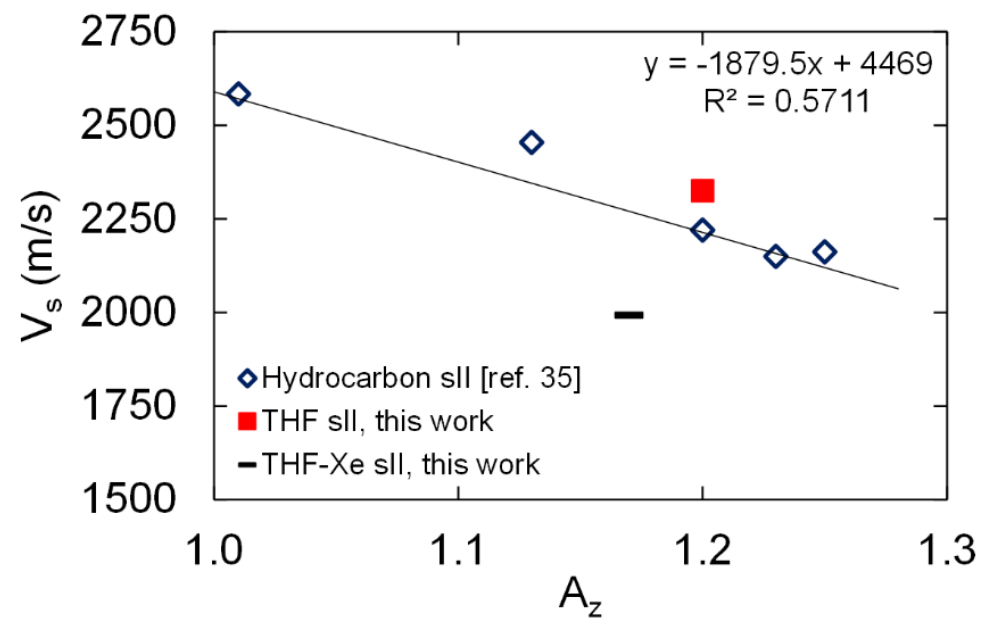

Figure S7. The effect of crystal anisotropy $\left(A_{z}\right)$ on the shear wave velocity.

SI 6. Atomic structure

Table S3. Our computed average equilibrium bond lengths $(\AA)$ and angles $\left(^{\circ}\right)$ for THF and THFxenon sll hydrates, compared to literature values for hydrocarbon sll hydrates.

\begin{tabular}{|c|c|c|c|c|c|c|}
\hline & THF & THF-Xe & Propane $^{a}$ & Isobutane $^{a}$ & $\begin{array}{l}\text { Ethane- } \\
\text { methane }{ }^{a}\end{array}$ & $\begin{array}{l}\text { Propane- } \\
\text { methane }^{a}\end{array}$ \\
\hline $\begin{array}{l}\mathrm{O}-\mathrm{H} \text { covalent } \\
\text { bond }\end{array}$ & 1.003 & 0.999 & 1.004 & 1.002 & 1.003 & 1.001 \\
\hline Hydrogen bond & 1.764 & 1.830 & 1.749 & 1.780 & 1.772 & 1.793 \\
\hline O-O distance & 2.766 & 2.827 & 2.752 & 2.781 & 2.773 & 2.793 \\
\hline $\mathrm{O}-\mathrm{O}-\mathrm{O}$ angles & 109.39 & 109.39 & 109.39 & 109.39 & 109.39 & 109.39 \\
\hline
\end{tabular}




\begin{tabular}{|l|c|c|c|c|c|c|}
\hline $\begin{array}{l}\text { Pentagonal face } \\
\text { angles }\end{array}$ & 107.98 & 107.99 & 107.99 & 107.98 & 107.99 & 107.99 \\
\hline $\begin{array}{l}\text { Hexagonal face } \\
\text { angles }\end{array}$ & 119.90 & 119.90 & 119.89 & 119.92 & 119.85 & 119.87 \\
\hline
\end{tabular}

avlasic et al. ${ }^{35}$

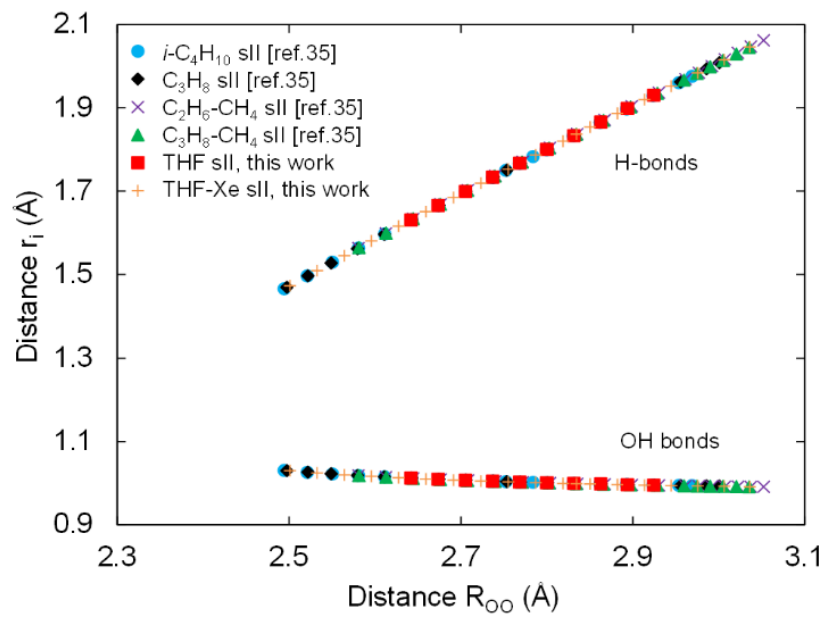

Figure S8. Bond length symmetrisation in sll hydrates.

Table S4. Hydrogen bond angles in THF and THF-xenon sll hydrates compared to sll hydrocarbon gas hydrates.

\begin{tabular}{|l|c|c|}
\hline Guest molecule(s) & $\mathrm{H}$-bond angle $\left(^{\circ}\right)$ & Standard deviation $\left(^{\circ}\right)$ \\
\hline THF, this work & 176.51 & 2.081 \\
\hline THF-Xe, this work & 176.55 & 2.134 \\
\hline $\mathrm{C}_{3} \mathrm{H}_{8}{ }^{a}$ & 176.70 & 2.000 \\
\hline$i-\mathrm{C}_{4} \mathrm{H}_{10}{ }^{a}$ & 176.57 & 2.066 \\
\hline $\mathrm{C}_{2} \mathrm{H}_{6}-\mathrm{CH}_{4}{ }^{a}$ & 176.72 & 1.971 \\
\hline $\mathrm{C}_{3} \mathrm{H}_{8}-\mathrm{CH}_{4}{ }^{a}$ & 176.72 & 2.031 \\
\hline
\end{tabular}

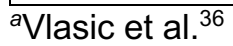

Table S5. Average large and small cage radii for sll hydrates at their equilibrium volume, illustrating cage stretching by the xenon atoms.

\begin{tabular}{|l|c|c|}
\hline Guest molecule(s) & Small cage radius $(\AA)$ & Large cage radius $(\AA)$ \\
\hline $\mathrm{C}_{3} \mathrm{H}_{8}$ & 3.841 & 4.638 \\
\hline$i-\mathrm{C}_{4} \mathrm{H}_{10}$ & 3.871 & 4.707 \\
\hline $\mathrm{C}_{2} \mathrm{H}_{6}-\mathrm{CH}_{4}$ & 3.884 & 4.649 \\
\hline $\mathrm{C}_{3} \mathrm{H}_{8}-\mathrm{CH}_{4}$ & 3.904 & 4.692 \\
\hline THF & 3.855 & 4.671 \\
\hline THF-Xe & 3.952 & 4.754 \\
\hline
\end{tabular}




\section{SI 7. Vibrational frequencies}

Table S6. Relevant THF and hydrocarbon hydrate literature values for the peak frequencies (in $\mathrm{cm}^{-1}$ ) of the $\mathrm{H}_{2} \mathrm{O}$ vibrational bands.

\begin{tabular}{|c|c|c|c|c|c|}
\hline Hydrate structure & $\begin{array}{l}\text { H-bond } \\
\text { stretch }\end{array}$ & $\begin{array}{c}\mathrm{H}_{2} \mathrm{O} \\
\text { libration }\end{array}$ & $\begin{array}{c}\mathrm{H}_{2} \mathrm{O} \\
\text { bending }\end{array}$ & $\begin{array}{c}\mathrm{OH} \text { symmetric } \\
\text { stretch }\end{array}$ & $\begin{array}{c}\mathrm{OH} \text { asymmetric } \\
\text { stretch }\end{array}$ \\
\hline${\text { THF sll, } 4 \mathrm{~K}, \exp ^{a}}^{a}$ & & & & 3076 & \\
\hline THF sll, $10 \mathrm{~K}$, exp. $^{b}$ & 235 & & & & \\
\hline${\text { THF sll, } 90 \mathrm{~K}, \text { exp. }^{c}}^{c}$ & 222 & & & 3092 & 3220 \\
\hline THF sll, $255 \mathrm{~K}$, exp. ${ }^{d}$ & 207 & & & 3144 & \\
\hline 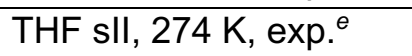 & & & & 3152 & 3354 \\
\hline $\mathrm{C}_{3} \mathrm{H}_{8}$ sll, $0 \mathrm{~K}$, theo. ${ }^{f}$ & 201 & 951 & 1632 & 3024 & 3240 \\
\hline$i-\mathrm{C}_{4} \mathrm{H}_{10}$ sll, $0 \mathrm{~K}$, theo. ${ }^{f}$ & 184 & 924 & 1634 & 3063 & 3275 \\
\hline $\mathrm{C}_{2} \mathrm{H}_{6}-\mathrm{CH}_{4}$ sll, $0 \mathrm{~K}$, theo. ${ }^{f}$ & 195 & 922 & 1631 & 3067 & 3260 \\
\hline $\mathrm{C}_{3} \mathrm{H}_{8}-\mathrm{CH}_{4}$ sll, $0 \mathrm{~K}$, theo. ${ }^{f}$ & 184 & 907 & 1631 & 3094 & 3285 \\
\hline $\mathrm{CH}_{4}$ sl, exp. ${ }^{g}$ & 206 & & & 3153 & 3369 \\
\hline
\end{tabular}

${ }^{a}$ Kato et al $.{ }^{55},{ }^{b}$ Tulk et al.${ }^{56}$, ${ }^{C}$ Prasad et al. ${ }^{57}$, dJohari and Chew ${ }^{58,59}$, ${ }^{6}$ Kumar et al. ${ }^{28}$, Vlasic et al. ${ }^{36}$, ${ }^{9}$ Schicks et al. ${ }^{60}$

Table S7. Relevant THF hydrate literature values for the band peak frequencies (in $\mathrm{cm}^{-1}$ ) of the guest vibrational modes.

\begin{tabular}{|l|c|c|c|c|c|}
\hline Hydrate structure & $\begin{array}{c}\mathrm{C}-\mathrm{C}-\mathrm{C}-\mathrm{C} \\
\text { stretch }\end{array}$ & $\begin{array}{c}\mathrm{C}-\mathrm{O}-\mathrm{C} \\
\text { stretch }\end{array}$ & $\begin{array}{c}\mathrm{CH}_{2} \\
\text { wag }\end{array}$ & $\begin{array}{c}\mathrm{CH}_{2} \\
\text { bend }\end{array}$ & $\mathrm{CH}_{2}$ stretch \\
\hline THF sll, 4 K, exp. ${ }^{a}$ & & & & & 2980 \\
\hline THF sll, 10 K, exp. ${ }^{b}$ & 925 & & & & $2863-2989$ \\
\hline THF sll, 13 K, exp. ${ }^{c}$ & & 1071 & & & \\
\hline THF sll, 90/240 K, exp. ${ }^{d}$ & $-/ 922$ & $-/ 1034$ & & & $2860-2985 /-$ \\
\hline THF sll, 240 K, exp..$^{p}$ & 916 & 1029 & 1232 & 1462 & $2879-2989$ \\
\hline THF sll, 274 K, exp..$^{f}$ & 918 & 1041 & 1236 & 1454 & $2877-2995$ \\
\hline THF sll, 275 K, exp. ${ }^{g}$ & 920 & 1033 & & & \\
\hline THF sll, 277 K, exp. ${ }^{h}$ & & 1036 & & & \\
\hline THF-C $_{2} \mathrm{H}_{6}$ sll, 277 K, exp. ${ }^{h}$ & 920 & 1036 & & & \\
\hline
\end{tabular}

${ }^{a}$ Kato et al. ${ }^{55},{ }^{b}$ Tulk et al. ${ }^{56}$, ${ }^{C}$ Fleyfel et al. ${ }^{61},{ }^{d}$ Prasad et al. ${ }^{57},{ }^{\mathrm{e}}$ Takasu et al. ${ }^{62},{ }^{\mathrm{f}} \mathrm{Kumar}$ et al. ${ }^{28}$, ${ }^{9}$ Carstensen et al. ${ }^{63},{ }^{h}$ Subramanian et al. ${ }^{64}$ 